\title{
Amination of anthra[1,9-cd:5,10-c,d,]bisisoxazole by alkylamines
}

\author{
Leonid M. Gornostaev*, Roman V. Mitrokhin, Oleg V. Podvyazny, and Elena V. Arnold \\ Department of Chemistry, Krasnoyarsk State Pedagogical University, Lebedevoy St. 89, \\ Krasnoyarsk, 660049, Russia \\ E-mail: gorn@edk.krasnoyarsk.su
}

(received 21 Dec 99; accepted 25 Apr 00; published on the web 03 May 00)

\begin{abstract}
Treatment of the stable analogue of a 1,5-anthraquinone, namely, anthra[1,9-cd:5,10$\mathrm{c}, \mathrm{d}$,]bisisoxazole, with primary and secondary amines in an inert atmosphere leads to the cleavage of one of the isoxazole moieties to afford 3(5)-alkylamino-7-amino-6H-6oxoanthra[1,9-cd]isoxazoles. However, amination in the presence of air leads to (5)-alkylaminoanthra[1,9-cd:5,10-c,d,]bisisoxazole without cleavage of the isoxazole moiety.
\end{abstract}

Keywords: Amination, anthrabisisoxazole

\section{Introduction}

Anthra[1,9-cd:5,10-c,d,]bisisoxazole is the only stable heteroanalogue of the thermo-dynamically unstable $^{1} 1,5$-anthraquinone (2). Few literature data exist on the reactivity of bisisoxazole (1). It is known that 6H-6-oxoanthra[1,9-cd] isoxazoles (3) (analogues of 1,10-anthraquinone) react by nucleophilic substitutions of hydrogen or halogens at positions 3,5. ${ }^{2}$ During the course of substitution of hydrogen in substrates such as (3), reductive cleavage of the $\mathrm{N}-\mathrm{O}$ bond in the isoxazole cycle frequently occurs. ${ }^{3}$

It is also known that boron complexes of 1,5-diamino- and 1,5-dihydroxy-9,10anthraquinones (4) have 1,5-anthraquinoid-like double bonds and, apparently because of this, undergo facile aminations. ${ }^{4}$ Obviously, this also accounts for the catalytic influence of cobalt salts upon amination of 1,5-diamino-9,10-anthraquinone by butylamine. ${ }^{5}$ 


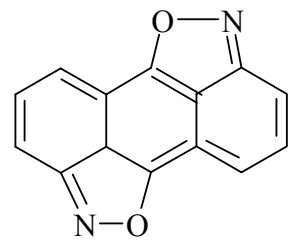

I<smiles>O=C1C=CC=c2cc3c(cc21)=CC=CC3=O</smiles>

II<smiles>O=C1c2ccccc2-c2onc3cccc1c23</smiles>

III<smiles></smiles>

IV

$\mathrm{X}=\mathrm{O}, \mathrm{NH} \quad \mathrm{Y}=\mathrm{OCOCH}_{3}$

On the basis of these observations, it was decided to investigate the reactions of bisisoxazole (1), as the most stable analogue of 1,5-anthraquinone, with alkylamines and to compare these reactions with those of 6H-6-oxoanthra[1,9-cd]isoxazoles (3) (analogues of 1,10-anthraquinone).

\section{Results and Discussion}

We have found that bisisoxazole (1) reacts with aliphatic and cyclic amines at room temperature in an inert atmosphere. The reaction products either crystallized spontaneously or were isolated by dilution of reaction mixture with water (see Table).

The reaction of bisisoxazole (1) with primary amines resulted in the formation of a mixture of substances, which were isolated by column chromatography. The aminations of substrate (1) by secondary amines proceeded in a much cleaner fashion.

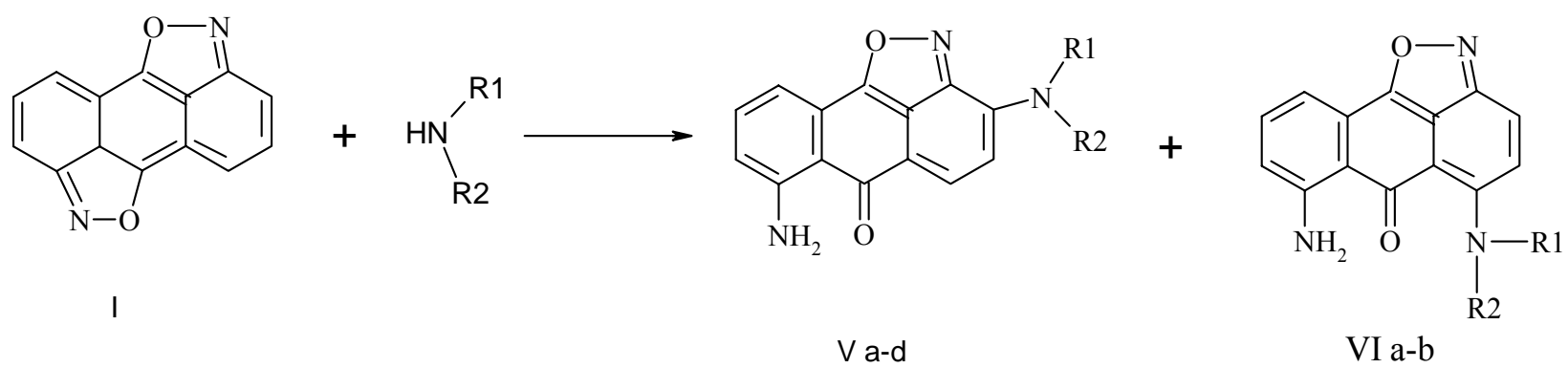

$\mathrm{V} \mathrm{R} 1=\mathrm{R} 2=\mathrm{CH}_{3}(\mathrm{a}) ; \mathrm{R} 1=\mathrm{H}, \mathrm{R} 2=\mathrm{n}-\mathrm{C}_{4} \mathrm{H}_{9}(\mathrm{~b}) ; \mathrm{R} 1 \mathrm{R} 2=\left(\mathrm{CH}_{2}\right)_{2} \mathrm{O}\left(\mathrm{CH}_{2}\right)_{2}(\mathrm{c}) ; \mathrm{R} 1 \mathrm{R} 2=\left(\mathrm{CH}_{2}\right)_{5}(\mathrm{~d})$ VI R1 $=\mathrm{H}, \mathrm{R} 2=\mathrm{n}-\mathrm{C}_{4} \mathrm{H}_{9}(\mathrm{a}) ; \mathrm{R} 1=\mathrm{H}, \mathrm{R} 2=\mathrm{CH}\left(\mathrm{CH}_{2}\right)_{5}(\mathrm{~b})$;

The structures of the 3(5)-alkylamino-7-amino-6H-6-oxoanthra[1,9-cd]isoxazoles (5-6) were confirmed by UV-, IR-, ${ }^{1} \mathrm{H}$ NMR-spectroscopic data and by their chemical transformations. For compounds (V-VI), vibrations of the NH-bond of primary amino group were observed at 3280$3440 \mathrm{~cm}^{-1}$ and vibrations of the $\mathrm{C}$ and vibrations of the $\mathrm{C}==\mathrm{O}$ bond were at $\mathrm{O}$ bond were at 
1635-1685 $\mathrm{cm}^{-1}$. Electronic absorption spectra of compounds (5-6) are similar to those of 3- or 5-alkylamino-6H-6-oxoanthra[1,9-cd]isoxazoles ${ }^{6}$ which have been previously reported.

The ${ }^{1} \mathrm{H}$ NMR spectra of isoxazoles (5-6) indicated the presence of a quinoid-type bonds, since the doublet signal for the proton at position 4 was found at higher field $(6.2-6.4 \mathrm{ppm})$. The broad singlet signal of protons of the primary amino group was centered in $\mathrm{CDCl}_{3}$ at $6.9-7.0 \mathrm{ppm}$.

The deamination of product Va led to 3-dimethylamino-6H-6-oxoanthra[1,9-cd]isoxazole (7a), which was shown to be identical to a previously synthesized sample. ${ }^{6}$ This fact confirmed the validity of structures 5-6.<smiles>CN(C)c1ccc2c3c(onc13)-c1cccc(N)c1C2=O</smiles>

$\mathrm{V}$ a<smiles>CN(C)c1ccc2c3c(onc13)-c1ccccc1C2=O</smiles>

VII a

The products of reduction of the isoxazolones $(5 \mathrm{~b}, 6 \mathrm{a})$ were identical to 1,5-diamino-2butylamino-9,10-anthraquinone (8a) and 1,5-diamino-4-butylamino-9,10-anthraquinone (8b), which were obtained independently according to known ${ }^{5}$ methods.<smiles>CCCCCNc1ccc2c3c(onc13)-c1cccc(N)c1C2=O</smiles>

$\mathrm{Vb}$

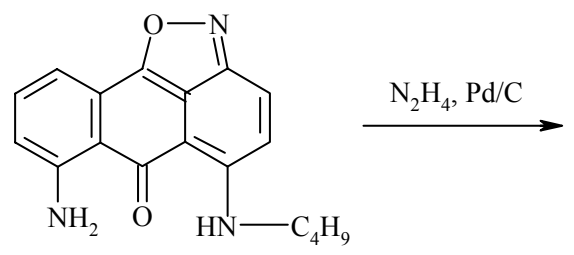

VI a<smiles>CCCCCCNc1ccc2c(c1N)C(=O)c1cccc(N)c1C2=O</smiles>

VIII a<smiles>CCCCCNc1ccc(N)c2c1C(=O)c1cccc(N)c1C2=O</smiles>

VIII b

The products (V-VI) were obtained via the following proposed pathway: 


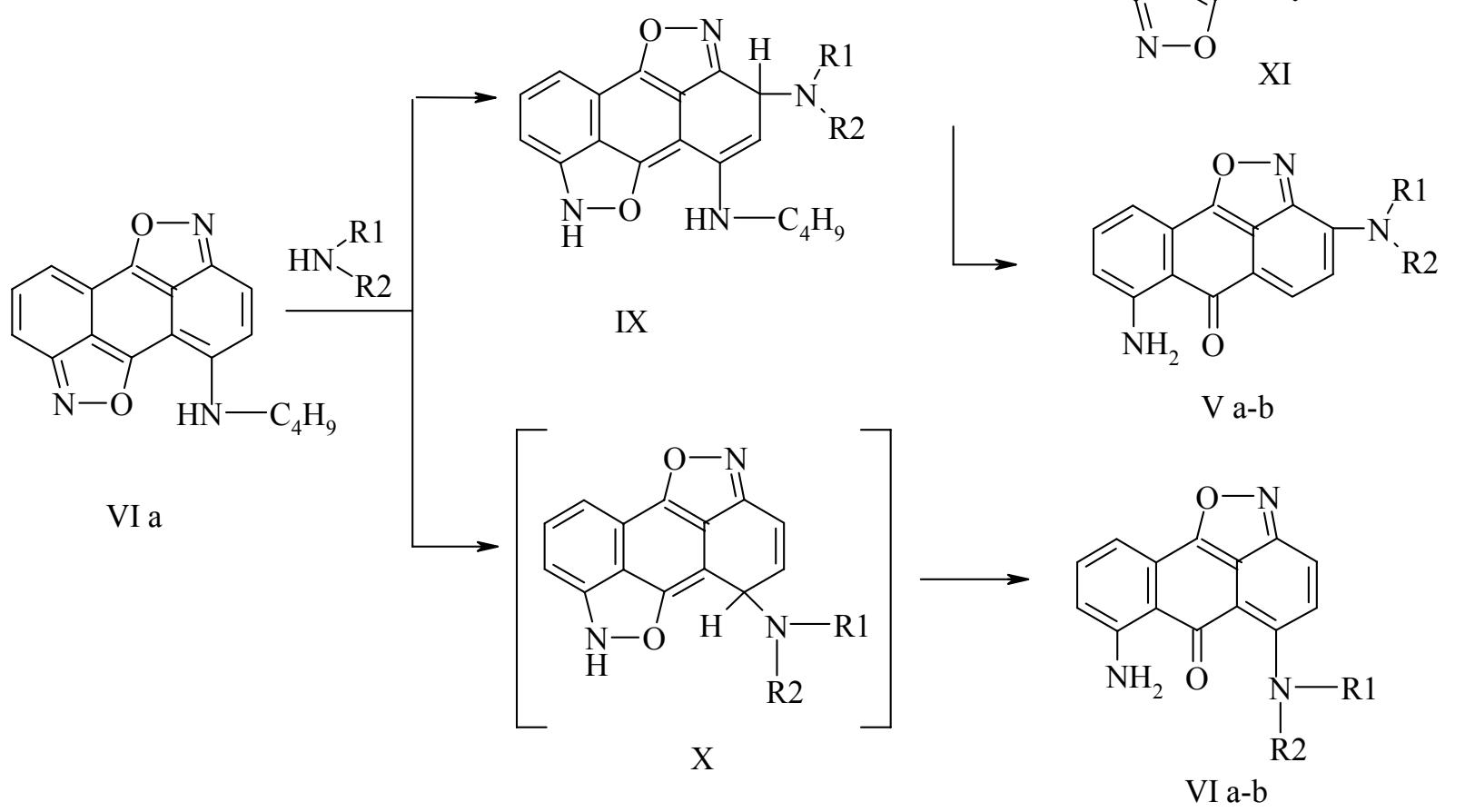

$\mathrm{V} \mathrm{R} 1=\mathrm{R} 2=\mathrm{CH}_{3}(\mathrm{a}) ; \mathrm{R} 1=\mathrm{H}, \mathrm{R} 2=\mathrm{n}-\mathrm{C}_{4} \mathrm{H}_{9}(\mathrm{~b}) ; \mathrm{R} 1, \mathrm{R} 2=\left(\mathrm{CH}_{2}\right)_{2} \mathrm{O}\left(\mathrm{CH}_{2}\right)_{2}$ (c); $\mathrm{R} 1, \mathrm{R} 2=\left(\mathrm{CH}_{2}\right)_{5}(\mathrm{~d})$; VI R1 $=\mathrm{H}, \mathrm{R} 2=\mathrm{n}-\mathrm{C}_{4} \mathrm{H}_{9}(\mathrm{a}) ; \mathrm{R} 1=\mathrm{H}, \mathrm{R} 2=\mathrm{CH}\left(\mathrm{CH}_{2}\right)_{5}(\mathrm{~b}) ; \mathrm{XI} \mathrm{R} 1=\mathrm{R} 2=\mathrm{CH}_{3}$

The reaction $(1 \rightarrow 5,6)$ studied by us differs from the previously reported reactions of reductive alkoxylation and amination of 6H-6-oxoanthra[1,9-cd]isoxazoles, heteroanalogues of 1,10anthraquinone [3,7], by the fact that the anthranyl fragment opposite to that is attacked is subjected to reductive cleavage in an inert atmosphere. In the presence of air the substrate (1) is transformed into 3-dimethylaminoanthra[1,9-cd:5,10-c,d,]bisisoxazole (9). 
Table 1. Constants, yields and data of elemental analyses of 3(5)-alkylamino-7-amino-6H-6oxoanthra[1,9-cd]isoxazoles

\begin{tabular}{|c|c|c|c|c|c|c|c|c|c|}
\hline \multirow{2}{*}{$\begin{array}{l}\text { Comp. } \\
\text { Number }\end{array}$} & \multirow[t]{2}{*}{$\mathrm{R}^{1}$} & \multirow[t]{2}{*}{$\mathrm{R}^{2}$} & \multirow{2}{*}{$\begin{array}{c}\text { Yield } \\
\% \\
\end{array}$} & \multirow{2}{*}{$\begin{array}{c}\text { Found } \\
\% \mathrm{~N} \\
\end{array}$} & \multirow[t]{2}{*}{ Formula } & \multirow{2}{*}{$\begin{array}{c}\text { Calculated } \\
\% \% \mathrm{~N}\end{array}$} & \multicolumn{2}{|c|}{$\begin{array}{c}\text { Electronic } \\
\text { absorp. } \\
\text { spectra } \\
\end{array}$} & \multirow[t]{2}{*}{$\mathrm{mp}{ }^{\circ} \mathrm{C}$} \\
\hline & & & & & & & $\lambda_{\max }$ & $\lg \varepsilon$ & \\
\hline \multirow[t]{3}{*}{$6 a$} & $\mathrm{H}$ & $\mathrm{HN}-\mathrm{n}-\mathrm{C}_{4} \mathrm{H}_{9}$ & 57 & 14.13 & $\mathrm{C}_{18} \mathrm{H}_{17} \mathrm{~N}_{3} \mathrm{O}_{2}$ & 13.68 & 240 & 4.5 & $152-154$ \\
\hline & & & & & & & 340 & 3.97 & \\
\hline & & & & & & & 550 & 4.26 & \\
\hline \multirow[t]{2}{*}{$5 b$} & $\mathrm{HN}-\mathrm{n}-\mathrm{C}_{4} \mathrm{H}_{9}$ & $\mathrm{H}$ & 25 & 13.68 & $\begin{array}{c}\mathrm{C}_{18} \mathrm{H}_{17} \\
\mathrm{~N}_{3} \mathrm{O}_{2}\end{array}$ & 13.60 & 235 & 5.16 & $160-162$ \\
\hline & & & & & & & 540 & 4.35 & \\
\hline \multirow[t]{3}{*}{$6 \mathrm{~b}$} & $\mathrm{H}$ & $\mathrm{HNCH}\left(\mathrm{CH}_{2}\right)_{5}$ & 60 & 12.97 & $\mathrm{H}_{20} \mathrm{H}_{19} \mathrm{~N}_{3} \mathrm{O}_{2}$ & 12.65 & 240 & 4.32 & $214-216$ \\
\hline & & & & & & & 340 & 3.9 & \\
\hline & & & & & & & 530 & 4.17 & \\
\hline \multirow[t]{2}{*}{$5 \mathrm{a}$} & $\mathrm{N}\left(\mathrm{CH}_{3}\right)_{2}$ & $\mathrm{H}$ & 70 & 15.03 & $\mathrm{C}_{16} \mathrm{H}_{13} \mathrm{~N}_{3} 0_{2}$ & 15.05 & 253 & 4.53 & $200^{\mathrm{a}}$ \\
\hline & & & & & & & 556 & 4.4 & \\
\hline \multirow[t]{2}{*}{$5 \mathrm{~d}$} & $\mathrm{~N}\left(\mathrm{CH}_{2}\right)_{5}$ & $\mathrm{H}$ & 46 & 12.99 & $\mathrm{C}_{19} \mathrm{H}_{17} \mathrm{~N}_{3} \mathrm{O}_{2}$ & 13.08 & 255 & 4.29 & $153-155$ \\
\hline & & & & & & & 560 & 4.3 & \\
\hline \multirow[t]{2}{*}{$5 c$} & $\mathrm{~N}\left(\mathrm{CH}_{2}\right)_{2} \mathrm{O}\left(\mathrm{CH}_{2}\right)_{2}$ & $\mathrm{H}$ & 50 & 13.00 & $\mathrm{C}_{18} \mathrm{H}_{15} \mathrm{~N}_{3} \mathrm{O}_{3}$ & 12.58 & 250 & 4.2 & $>300$ \\
\hline & & & & & & & 540 & 4.16 & \\
\hline
\end{tabular}

${ }^{\mathrm{a}}$ Decomposes while melting.

\section{Experimental Section}

General Procedures. Electronic absorption spectra were recorded in ethanol on an SF-26 spectrometer. The IR spectra were recorded in $\mathrm{KBr}$ pellets on a Specord 75 IR spectrometer. The ${ }^{1} \mathrm{H}$ NMR spectra were recorded on a Bruker AM 400 spectrometer. All chemical shifts are given relative to $\mathrm{SiMe}_{4}$ as internal standard and for $\mathrm{CDCl}_{3}$ as a solvent. Melting points were determined on a Boetius melting-point apparatus. The progress of the reactions was ascertained by thin-layer chromatography on Silufol plates; eluent: toluene-acetone-hexane 10:1:1.

Synthesis. Anthra[1,9-cd:5,10-c,d,]bisisoxazole (1) was obtained according to the described methods. $^{8}$ Aminations of anthra[1,9-cd:5,10-c,d,]bisisoxazole (1). . Bisisoxazole (1) 
$(0.025 \mathrm{~mol})$ was added to DMAA $(20 \mathrm{~mL})$ at $20-25{ }^{\circ} \mathrm{C}$. This mixture was saturated with argon. While stirring, $2 \mathrm{~mL}$ of $30 \%$ water solution of dimethylamine (or $2 \mathrm{~mL}$ of another amine such as butylamine, piperidine, morpholine, or cyclohexylamine) were added. The mixture was kept at $20-25{ }^{\circ} \mathrm{C}$ for $20-80 \mathrm{~h}$ during which time the solution acquired a violet coloration. The precipitate of 7-amino-3-dimethylamino-6H-6-oxoanthra[1,9-cd]isoxazole (5a) was collected by filtration and washed with ethanol. The other isoxazolones were isolated in the following way: the reaction mixture was heated to $50-60{ }^{\circ} \mathrm{C}$, the solution was diluted with water $(5-15 \mathrm{~mL})$ and cooled to $0 \pm 5{ }^{\circ} \mathrm{C}$. The obtained precipitate was collected by filtration, dried and purified by column chromatography (adsorbent: $\mathrm{Al}_{2} \mathrm{O}_{3}$; eluent: toluene). After removal of the solvent, the products $(5 \mathrm{~b}, \mathrm{c}, \mathrm{d}, 6)$ were characterized.

7-Amino-3-dimethylamino-6H-6-oxoanthra[1,9-cd]isoxazole (5a). ${ }^{1} \mathrm{H}$ NMR $\delta 3.5(6 \mathrm{H}$, s, $\left.\mathrm{N}\left(\mathrm{CH}_{3}\right)_{2}\right), 6.21(1 \mathrm{H}, \mathrm{d}, \mathrm{H}-4), 6.71(1 \mathrm{H}, \mathrm{d}, \mathrm{H}-5), 7.29(1 \mathrm{H}, \mathrm{d}, \mathrm{H}-8), 7.33$ (1H, t, H-9), $7.81(1 \mathrm{H}$, d, H-10).

7-Amino-3-(N-morpholino)-6H-6-oxoanthra[1,9-cd]isoxazole (5c). ${ }^{1} \mathrm{H}$ NMR $\delta 6.45$ (1H, d, H-4), 6.74 (1H, d, H-8), 7.00 (2H, s, NH 2$), 7.30$ (1H, d, H-10), 7.34 (1H, t, H-9), 7.78 (1H, d, H-5).

7-Amino-6H-6-oxo-3-( $N$-piperidino)anthra[1,9-cd]isoxazole (5d). ${ }^{1} \mathrm{H}$ NMR $\delta 6.41$ (1H, d, H-4), 6.67 (1H, d, H-8), 6.98 (2H, s, NH $), 7.29$ (1H, d, H-10), 7.38 (1H, t, H-9), 7.79 (1H, d, H-5).

7-Amino-5-butylamino-6H-6-oxoanthra[1,9-cd]isoxazole (6a). ${ }^{1} \mathrm{H}$ NMR $\delta 0.99(3 \mathrm{H}, \mathrm{t}, \mathrm{Bu})$, $1.56(2 \mathrm{H}, \mathrm{m}, \mathrm{Bu}), 1.76(2 \mathrm{H}, \mathrm{m}, \mathrm{Bu}), 3.52\left(2 \mathrm{H}, \mathrm{m}, \mathrm{N}-\mathrm{CH}_{2}\right), 6.68(1 \mathrm{H}, \mathrm{d}, \mathrm{H}-4), 6.29(2 \mathrm{H}, \mathrm{s}$, $\left.\mathrm{NH}_{2}\right), 7.24$ (1H, d, H-10), 7.32 (1H, t, H-9), 7.39 (1H, d, H-8), 7.74 (1H, d, H-3), 9.81 (1H, br, $\mathrm{NH})$.

7-Amino-5-cyclohexylamino-6H-6-oxoanthra[1,9-cd]isoxazole (6b). ${ }^{1} \mathrm{H}$ NMR $\delta 6.68(1 \mathrm{H}, \mathrm{d}$, H-3), 6.85 (2H, s, NH$\left.)_{2}\right), 7.23$ (1H, t, H-9), 7.3 (1H, d, H-8), 7.35 (1H, d, H-10), 7.73 (1H, d, H4), $9.92(1 \mathrm{H}, \mathrm{br}, \mathrm{NH})$.

3-Dimethylamino-6H-6-oxoanthra[1,9-cd]isoxazole (7a). The 3-chloro-6H-6-oxoanthra[1,9$c d$ ] isoxazole $(0.008 \mathrm{~mol})$ was added to methanol $(5 \mathrm{~mL})$. While stirring dimethylamine $(0.04 \mathrm{~mol})$ was added and the mixture was heated to $30-35{ }^{\circ} \mathrm{C}$. Upon completion of the reaction, VIIa was collected by filtration and washed with ethanol. The product was purified by column chromatography (adsorbent: silica gel; eluent: benzene); yield 46\%; mp 164-166 ${ }^{\circ} \mathrm{C}$; Rf 0.88 (eluent: acetone; Silufol UV 254 plates).

Deamination of 7-amino-3-dimethylamino-6H-6-oxoanthra[1,9-cd]isoxazole (5a). A freshly prepared suspension of cuprous oxide $(0.8 \mathrm{~g})$ in absolute ethanol $(20 \mathrm{~mL})$ was added to a solution of $\mathrm{Va}(0.002 \mathrm{~mol})$ in acetic acid $(20 \mathrm{~mL})$. While being kept at room temperature, sodium nitrite $(0.3 \mathrm{~g}, 0.0042 \mathrm{~mol})$ was added by portions. The mixture was heated slowly to $50{ }^{\circ} \mathrm{C}$ and kept at this temperature during $20 \mathrm{~min}$ Water $(5-15 \mathrm{~mL})$ was added to the hot filtrate and the mixture was cooled to $0-+5{ }^{\circ} \mathrm{C}$. The precipitate was collected by filtration and 
washed with ethanol. The product was chromatographed on silica gel with benzene as the eluent; yield 64\%; mp 164-166 ${ }^{\circ} \mathrm{C}$; Rf 0.88 (eluent: acetone; Silufol UV 254 plates).

3-Dimethylaminoanthra[1,9-cd:5,10-c,d,]bisisoxazole (9). Bisisoxazole (1) (0.005 mol) was dissolved in DMFA $(40 \mathrm{~mL})$ and a $30 \%$ aqueous solution of dimethylamine $(10 \mathrm{~mL})$ was added. The mixture was stirred at $0-+10$ for $72 \mathrm{~h}$ and the mixture was cooled. The precipitate of XI was collected by filtration and washed with ethanol; yield $60 \%$; mp $132{ }^{\circ} \mathrm{C}$ (decomp.); $\lambda_{\max } 600 \mathrm{~nm}$, (Found: N, 15.03. Calc.: N, 15.05); ${ }^{1} \mathrm{H}$ NMR $\delta 3.49\left(6 \mathrm{H}, \mathrm{s}, \mathrm{N}\left(\mathrm{CH}_{3}\right)_{2}\right), 6.28(1 \mathrm{H}$, d, H-4), 7.23 (1H, d, H-5), 7.32 (1H, d, H-10), 7.41 (1H, t, H-9), 7.48 (1H, d, H-8).

Reduction of 7-amino-3-butylamino- (5b) and 7-amino-5-butylamino-6H-6oxoanthra[1,9-cd]isoxazoles (6a). Butylamino derivative (5b or $6 \mathrm{a})(0.1 \mathrm{~g})$ was boiled in ethanol $(20 \mathrm{~mL})$ with hydrazine hydrate $(1 \mathrm{~mL})$ in the presence of $\mathrm{Pd} / \mathrm{C}(0.1 \mathrm{~g})$. Upon completion of the reaction, the hot reaction mixture was filtered .The product of the reduction ( $8 \mathrm{a}$ or $8 \mathrm{~b}$, respectively) was isolated by dilution of the filtrate with water.

2-Butylamino-1,5-diaminoanthraquinone (8a). ${ }^{1} \mathrm{H}$ NMR $\delta_{\mathrm{H}}\left(\mathrm{DMSO}_{-} \mathrm{d}_{6}\right) 0.94\left(3 \mathrm{H}, \mathrm{t},-\mathrm{CH}_{3}\right)$, $1.53\left(4 \mathrm{H}, \mathrm{m},-\left(\mathrm{CH}_{2}\right)_{2}-\right), 3.20\left(2 \mathrm{H}, \mathrm{t}, \mathrm{N}-\mathrm{CH}_{2}-\right), 5.92(1 \mathrm{H}, \mathrm{br}, \mathrm{NH}), 6.6-7.6(5 \mathrm{H}, \mathrm{m}, \mathrm{H}-3,-4,-6,-7$ and $\mathrm{H}-8), 7.74\left(2 \mathrm{H}, \mathrm{br}, \mathrm{NH}_{2}\right), 7.98\left(2 \mathrm{H}, \mathrm{br}, \mathrm{NH}_{2}\right)$.

4-Butylamino-1,5-diaminoanthraquinone (8b). ${ }^{1} \mathrm{H}$ NMR $\delta_{\mathrm{H}}\left(\mathrm{DMSO}_{6}\right) 0.94\left(3 \mathrm{H}, \mathrm{t},-\mathrm{CH}_{3}\right)$, $1.53\left(4 \mathrm{H}, \mathrm{m},-\left(\mathrm{CH}_{2}\right)_{2}-\right), 3.36\left(2 \mathrm{H}, \mathrm{q}, \mathrm{N}-\mathrm{CH}_{2}-\right), 6.9-7.5(5 \mathrm{H}, \mathrm{m}, \mathrm{H}-2,-3,-6,-7$ and $\mathrm{H}-8), 7.71$ $\left(2 \mathrm{H}, \mathrm{br}, \mathrm{NH}_{2}\right), 8.18\left(2 \mathrm{H}, \mathrm{br}, \mathrm{NH}_{2}\right), 10.36(1 \mathrm{H}, \mathrm{br}, \mathrm{NH})$.

\section{Acknowledgements}

This work has been done under the financial support of Krasnoyarsk Regional Science Foundation, grant 7F0187, 8F0102.

\section{References}

1. Gorelik, M. V. Khimiya antrakhinonov i ikh proizvodnikh, Khimiya: Moscow 1983; p. 78.

2. Galushko, A. M.; Dokunikhin, N. S. Zh. Org. Khim. 1982, 18, 1539.

3. Eskin, A. P.; Gornostaev, L. M.; Zeibert, G. F.; Eltsov, A. V. Zh. Org. Khim. 1990, 26, 188.

4. Gorelik, M. V.; Shapetko, N. N.; Arinich, L. V.; Tsurkan, A. I.; Kukushkina, M. L. Zh. Org. Khim. 1986, 22, 611.

5. Katsuhira Yoshida; Takashi Fujiwara; Yoshio Yamashita Chem. Express 1986, 1, 595.

6. Gornostaev, L. M.; Zeibert, G. F.; Zolotareva, G. I. Khim. Geterotsikl. Soed. 1980, 7, 912.

7. Galushko, A. M.; Dokunikhin, N. S. Zh. Org. Khim. 1979, 15, 575. 
8. Gattermann, L.; Rolfes, H. Ann. 1921, 425, 135. 